\title{
Frequency of the Common MYH Mutations (G382D and Y165C) in MMR Mutation Positive and Negative HNPCC Patients
}

\author{
Katie A. Ashton', Cliff J. Meldrum², Mary L. McPhillips', Carla F. Kairupan', Rodney J. Scott ${ }^{1,2}$ \\ IDiscipline of Medical Genetics, School of Biomedical Sciences, Faculty of Health, University of Newcastle and the Hunter Medical Research Institute, Newcastle, New \\ South Wales, Australia; 2Division of Genetics, Hunter Area Pathology Service, John Hunter Hospital, Newcastle, New South Wales, Australia
}

Key words: HNPCC, mutations, MYH, modifier genes, MMR, BER

Corresponding author: Rodney J. Scott, Faculty of Health, University of Newcastle NSW 2308 and the Hunter Medical Research Institute Newcastle, Australia,e-mail: rodney.scott@newcastle.edu.au

Submitted: 3 May 2005

Accepted: 10 May 2005

\begin{abstract}
Recently mutations in the MYH gene have been associated with a milder form of adenomatous polyposis which is characterized by a variable level of colonic polyps ranging from a few to several hundred. In the context of HNPCC it is not unusual to identify patients with a smattering of polyps. The MYH gene product is involved in DNA repair and indeed the hMSH2/hMSH6 complex (both genes being essential elements of the DNA mismatch repair pathway) is required to stimulate $\mathrm{MYH}$ activity. We reasoned that because of the clinical similarity of a subset of HNPCC patients to those described with MYH mutations and the role of the hMSH2/hMSH6 complex in the activation of MYH protein that MYH mutations may account for a small proportion of HNPCC patients. In a study of 442 HNPCC patients we identified MYH mutations at the same frequency as that expected in the general population. Nevertheless, two HNPCC families were identified harbouring biallelic changes in $M Y H$.
\end{abstract}

\section{Introduction}

Hereditary Non Polyposis Colorectal Cancer (HNPCC) is an autosomal dominant inherited disorder associated with a familial predisposition to colorectal cancer (CRC). It is characterised by early age of disease onset, neoplastic lesions with microsatellite instability (MSI) and an increased incidence of extracolonic malignancies such as cancers of the endometrium, ovary, stomach, small bowel, ureter and renal pelvis [1]. Approximately $20 \%$ of all CRC cases display familial inheritance [2] however familial colorectal cancer syndromes, that include HNPCC and FAP account for around $3 \%$ of all colorectal cancer patients [3]. A significant number of cases that appear to be inherited therefore do not have mutations in known genes involved in familial cancer syndromes. HNPCC has been found to be associated with hMLH 1 and hMSH2 genes involved in the mismatch repair (MMR) pathway. Germline mutations in hMLH1 and hMSH2, the main causes of mismatch repair deficiency, have been linked with a significantly increased risk for developing HNPCC $[4,5]$. Among all HNPCC patients only $80 \%$ of men and $40 \%$ of women that have germline mutations in MMR genes develop CRC $[6,7]$ and a further $25-50 \%$ of women develop endometrial cancer [8]. MMR gene mutations do not account for all CRC cases in HNPCC since some patients fit the clinical criteria for HNPCC yet they do not harbour a MMR gene mutation. Therefore, 
it is highly likely that there are other genes involved in the aetiology of HNPCC.

MYH has recently been identified as an autosomal recessive inherited form of colonic polyposis. It functions to repair DNA damage caused by reactive oxygen species through the DNA repair process known as base excision repair (BER). MYH removes adenines misincorporated into DNA opposite guanine or 7,8-dihydro-8-oxo-guanine to prevent G:C to T:A mutations (for review see [9]). Al-Tassan et al [10] were the first to describe the role of $\mathrm{MYH}$ in colorectal polyposis and since then a number of studies have confirmed this association [1 1-17]. The results obtained in these studies suggest that colorectal cancer and in particular HNPCC might not only be confined to mutations in MMR genes but also to other genes involved in DNA repair such as $\mathrm{MYH}$.

Two known polymorphisms in MYH, Y165C and G382D, are functionally compromised in the variant form. Al-Tassan et al [10] demonstrated that the variant forms of these proteins reduced the glycosylase ability of the proteins to remove DNA damage caused by reactive oxygen species (8-oxoG:A). A study by Jones et al [15] showed that biallelic nonsense and missense mutations in $\mathrm{MYH}$ were found in colorectal cancer patients that were unrelated and in addition they identified that sequence variants are found in different ethnic groups that include the Caucasian (Y165C, G382D), Indian (E466X), Pakistani (Y90X) and the Italian (1395delGGA). It is not currently known whether MYH mutations in the heterozygous form cause CRC or if they modify the effects of genes already associated with CRC such as APC, hMLH1 or hMSH2. The study performed by Kairupan et al [11] demonstrated that MYH mutations Y165C, G382D and 1391delAGG were not present in the APC mutation positive FAP population which suggests that $\mathrm{MYH}$ is not a modifier gene in FAP.

It has recently been shown that the hMSH2/hMSH6 heterodimer formed as part of the MMR process functions to stimulate the activation of $M Y H$ thereby linking two DNA repair processes and implying a connection of MYH to HNPCC [18]. Gu et al [18] suggest that hMSH proteins and $M Y H$ have a combined effect to remove any DNA damage that occurs. They have shown a direct interaction between the hMSH2/hMSH6 heterodimer between hMSH6 and MYH at residue 232-254 of MYH. Mutant MYH has also been shown to have a decreased ability to recognize and repair mismatches that occur due to reactive oxygen species (ROS). Additionally, Gu et al
[18] showed that MSH2 defective cells have an impaired function in oxidative damage. This shows that MSH2 and MYH both have roles in the removal of oxidative damage. They suggest that this interaction must be studied further to determine whether the mutant forms of MYH disrupt the MMR and BER pathways in colorectal tumourigenesis. Single nucleotid polymorphisms (SNPs) found in MYH could potentially interfere with these protein-protein interactions thereby hindering the ability of MMR and BER pathways to remove DNA damage and consequently promoting colorectal cancer [12].

Kambara et al [13] suggest that there are a number of other tumour suppressors on chromosome $1 p$ since microsatellite stable (MSS) colorectal tumours often show loss of heterozygosity $(\mathrm{LOH})$ on chromosome 1 , however $\mathrm{LOH}$ of chromosome 1 is not usually evident within patients that have high microsatellite instability (MSI-H) tumours. MSI-H tumours are usually associated with HNPCC. This suggests that dysfunction of tumour suppressors on chromosome 1 might display a different phenotype to that of HNPCC (microsatellite stabile [MSS] but a small number of adenomas).

In the current study, we have investigated a series of patients that have been extensively screened for $h M L H 1$ and hMSH2 germline mutations by denaturing high performance liquid chromatography (dHPLC) analysis, denaturing gradient gel electrophoresis (DGGE), direct sequencing and multiplex ligation probe amplification (MLPA) analysis. Using this screening strategy, two groups of patients were identified: patients with a confirmed germline mutation in $h M L H 1$ or hMSH2, and patients that were mutation negative. These two groups were assessed for the presence of the two common MYH mutations (Y165C and G382D) to determine whether patients that manifest the clinical characteristics of HNPCC harbour these mutations.

\section{Methods}

\section{Patients}

Patients were selected from across the State of New South Wales because they fulfilled the clinical criteria of Hereditary Non Polyposis Colorectal Cancer (HNPCC). All patients enrolled in this study had given informed consent for their anonymous DNA to be used for research into genetic predispositions to colorectal cancer. This study was carried out with the approval of the Hunter Area and the University of Newcastle's Research Ethics Committees. 
Table 1. Genetic alterations identified in the MYH gene and disease characteristics of the patients

\begin{tabular}{|c|c|c|c|c|c|c|c|c|}
\hline $\begin{array}{l}\text { Patient } \\
\text { Number }\end{array}$ & $\begin{array}{c}\text { HNPCC } \\
\text { MMR } \\
\text { Mutation }\end{array}$ & $\begin{array}{c}\text { First } \\
\text { Alteration }\end{array}$ & $\begin{array}{c}\text { Second } \\
\text { Alteration }\end{array}$ & Sex & $\begin{array}{c}\text { Age of } \\
\text { Diagnosis } \\
\text { of Adenomas }\end{array}$ & $\begin{array}{c}\text { Number } \\
\text { of Adenomas }\end{array}$ & $\begin{array}{c}\text { CRC } \\
\text { (Age of Diagnosis) }\end{array}$ & $\begin{array}{l}\text { Other } \\
\text { Cancers }\end{array}$ \\
\hline 1 & NO & Y165C & NO & $\mathrm{F}$ & 43 & 1 & N-AF & \\
\hline 2 & NO & G382D & V22M & $\mathrm{F}$ & $>30$ & $?$ & $\mathrm{AF}(36)$ & \\
\hline 3 & hMSH2 $(\mathrm{P})^{*}$ & G382D & NO & M & 46 & 3 & $A F(46)$ & Melanoma (42) \\
\hline 4 & NO & G382D & NO & $M$ & $>70$ & $?$ & $\mathrm{~N}-\mathrm{AF}$ & Gastric (78) \\
\hline 5 & hMSH2 & G382D & NO & $M$ & $>30$ & $?$ & N-AF & \\
\hline 6 & NO & G382D & V22M & $\mathrm{F}$ & $>40$ & $?$ & $\operatorname{AF}(47)$ & \\
\hline 7 & NO & G382D & NO & $\mathrm{F}$ & 45 & 1 & N-AF & \\
\hline
\end{tabular}

* hMSH2 polymorphism with an unknown functional consequence

The patients involved in this study fulfilled either the Amsterdam or Bethesda HNPCC criteria. This study included patients from families that fit the clinical criteria for HNPCC even though they might not have developed disease nor had a mutation in $\mathrm{hMLH} 1$ and hMSH2. Three groups of patients were used in this study. Group 1 consisted of 233 patients of which 162 patients were affected with CRC who were shown not to harbour germline mutations in the $h M L H 1$ and hMSH2 genes by denaturing high performance liquid chromatography (dHPLC) analysis followed by direct sequencing, the multiplex ligation probe amplification (MLPA) assay and denaturing gradient gel electrophoresis (DGGE). This group of patients was collected from 1997 to 2004. Group 2 consisted of 209 patients that had a confirmed mutation in either hMLH1 or hMSH2. This group of patients was collected from 1997 to 2004. Group 3 (control group) consisted of a series of 296 anonymous control DNA samples collected between the years of 1993 and 1997 with a mean age of 51 years (range 30-94).

\section{DNA isolation}

Genomic DNA was isolated from $\mathrm{Na}_{2}$ EDTA blood according to the method previously described by Miller et al [19].

\section{Real Time PCR (RT-PCR) SNP genotyping}

DNA samples were SNP genotyped to determine the allele frequency of the two common MYH mutations, Y165C and G382D. Allelic discrimination was performed on an ABI PRISM ${ }^{\circledast} 7900 \mathrm{HT}^{2}$ sequencing detection system (PE Applied Biosystems, Foster City, USA). Assay-by-Design ${ }^{\text {SM }}$, a service that is offered by
Applied Biosystems (PE Applied Biosystems, Foster City, USA), was used to design primers and probes. The assay functions under universal reactions and conditions with each reaction containing: 50ng DNA, $0.125 \mu \mathrm{l}$ 40x Assay Mix, $2.5 \mu \mathrm{l}$ Taqman $^{\oplus}$ Universal PCR master mix made up to $5 \mu \mathrm{l}$ with sterile MilliQ water. The thermal cycling conditions were: $50^{\circ} \mathrm{C}$ for 2 minutes, $95^{\circ} \mathrm{C}$ for 10 minutes, and 40 cycles of $92^{\circ} \mathrm{C}$ for 15 seconds and $60^{\circ} \mathrm{C}$ for 1 minute. Post PCR, the plate was scanned to allow discrimination between the different genotypes.

\section{Primer and probe sequences}

The primers and probes used for genotyping both MYH mutations are listed below:

\section{Y165C}

Forward

Reverse

Wild type probe

Mutant probe

\section{5'-CCACAGGAGGTGAATCAACTCT}

5'-CCTTACCTTCCGAGCTCCCT

VIC-CTGGGCTACTATTCT

FAM-TGGGCTGCTATTCT

\section{G382D}

Forward

Reverse

Wild type probe

Mutant probe

\section{5' - GACCCCTGCCTGGCT \\ 5' - GACGGGAACTCCCACAGT \\ VIC - CCTCTCAGGTCTGCTG \\ FAM - CCTCTCAGATCTGCTG}

\section{DNA sequencing of the MYH gene}

All patients who were determined to be heterozygous for either Y165C or G382D polymorphisms were further investigated for additional mutations in the MYH gene. Primers designed by Kairupan et al [1 1] were used for this analysis. 
DNA analysis was performed using a semiautomated sequencing unit (model 310, Perkin-Elmer Applied Biosystems Division, Foster City, USA) according to the manufacturer's instructions. All germline mutations identified were confirmed by reverse sequencing. The sequence variant position in the MYH gene was compared to that on the NCBI database, accession number BC003178.1.

\section{Statistics}

Differences in allele frequency between groups were determined by chi-squared analysis. Statistical differences were assessed using the STATA statistical package (STATA Corporation, College Station TX); $p$ values less than 0.05 were considered significant.

\section{Results}

In total, 442 HNPCC patients were assessed for the presence of two MYH polymorphisms, Y165C and G382D. The frequency of the mutations in the entire HNPCC group compared to the controls was not significant $(p=0.798)$.

In the control group of 296 patients, the G382D mutation was found at a frequency of $1.35 \%$ and the Y165C mutation was not found in any of the controls. The frequency observed in the control population is consistent with the observed frequency in other populations studied.

In group 1 (mutation negative), of the 233 patients, 1 was heterozygous for the $\mathrm{Y} 165 \mathrm{C}$ mutation and 4 were heterozygous for the G382D mutation. The frequency of the mutations in this group was not significantly different as compared to group $2(p=0.317)$ or the control population $(p=0.483)$.

In group 2 (mutation positive), of the 209 patients, 2 were heterozygous for the G382D mutation. One patient had a confirmed causative mutation in hMSH2 and the other had 2 polymorphisms, one of which was non-causative as judged by information on the Insight mutation database (www.insight-group.org) and the other was an unknown functional variant $(1661+12 A>G$ and $1551-9 T>A$, respectively). The polymorphism in intron 9 is close to the intron-exon boundary of intron 9 and exon 10 and therefore could possibly have a detrimental functional consequence. The frequency of the mutations in this group was not significantly different as compared to the controls $(p=0.687)$.
The DNA of all patients that were heterozygous for Y165C and G382D was further analysed to determine whether any other mutations were present. Two of the seven patients were heterozygous for the G382D polymorphism, harboured the causative mutation V22M. No other changes were found.

\section{Discussion}

Identifying genes involved in colorectal cancer remains a challenge since known mutations in inherited disorders only account for approximately $2-5 \%$ of all CRC cases [5]. A large proportion of CRC cases that appear to have Mendelian dominant inheritance, however, have an unknown genetic cause of disease. It has been suggested that inherited predispositions to CRC are possibly due to low-penetrance genetic variants clustered at many loci [20].

In our study, we evaluated the two common $\mathrm{MYH}$ polymorphisms in the Caucasian population, Y165C and G382D, which are associated with an increased risk for developing CRC, in an Australian series of HNPCC patients. The MYH polymorphisms studied previously have been found in $1 \%$ of the general population and the findings in the current study confirm this result since $1.35 \%$ of the control population was heterozygous for $\mathrm{MYH}$ mutations, all were heterozygous for G382D.

In total 7 HNPCC patients were heterozygous for 1 of the 2 polymorphisms in MYH (1.6\%). Five of the patients were from the sub-population of HNPCC patients who were shown not to harbour mutations in the DNA mismatch repair genes $h M S H 2$ or $h M L H 1$. This accounts for approximately $2 \%$ of the HNPCC mutation negative population. Of the five $\mathrm{MYH}$ mutation carriers, one patient was heterozygous for Y165C and four patients were heterozygous for G382D. Direct sequencing of the MYH gene revealed that two of them harboured the V22M mutation, which has been previously identified [11, 13, 21, 22]. Kairupan et al [1 1] showed that 1 patient heterozygous for the Y165C mutation also harboured the V22M polymorphism. Together, the evidence suggests that the V22M polymorphism could be an additional common $\mathrm{MYH}$ polymorphism associated with colorectal cancer. The suggestion that there are other common MYH polymorphisms associated with colorectal cancer needs to be verified in a much larger study. In the remaining 5 patients no evidence of a second mutation was forthcoming but the possibility remains that mutations could be present in the 
promoter region of $\mathrm{MYH}$. Alternatively, exon deletions cannot be ruled out as these were not investigated.

Approximately $1 \%$ of the HNPCC mutation positive population carried the MYH mutations. Two patients that were heterozygous for the G382D mutation harboured hMSH2 mutations, one of which has not been functionally evaluated. This is the first study to describe the existence of MYH polymorphisms in association with MMR mutation positive patients. This is an interesting finding since the MMR heterodimer hMSH2/hMSH6 interacts directly with MYH through hMSH6 to stimulate DNA binding and glycosylase activity to remove mismatches caused by reactive oxygen species (ROS) which are the most prevalent source of DNA damage in aerobic organisms $[12,18]$. A previous study suggests that MYH does not initiate progression of cancer but it acts as a promoter [13].

In a previous study, Kairupan et al [11] found that the MYH mutations, Y165C and G382D, were not represented in FAP APC mutation positive patients suggesting that $M Y H$ does not play a modifying role in APC driven FAP. Interestingly, this study shows that MYH mutations could possibly modify the functionality of the heterodimer hMSH2/hMSH6 since two patients with mutations in $\mathrm{hMSH} 2$ were also heterozygous for $\mathrm{MYH}$ (G382D) mutations.

In addition, MYH mutations were found in the HNPCC MMR mutation negative group. This result suggests that MYH may account for some of the cases of CRC where no genetic predisposition has been identified. In a previous study we have shown that the clinical features of some of the patients harbouring MYH mutations are similar to HNPCC patients [1 1]. In this study, we found that from the clinical information available on each patient only a few adenomas were identified in patients harbouring mutations in $\mathrm{MYH}$.

Similar to Kairupan et al [11], in the HNPCC population studied herein there was no overrepresentation of extracolonic cancers apart from one patient diagnosed with melanoma and another with gastric cancer. Together the evidence suggests that upper gastrointestinal malignancies might not be a feature of this type of colorectal cancer predisposition.

In conclusion, the results of our study show that MYH is associated with colorectal cancer risk and can possibly be associated with the clinical features of HNPCC. This finding may aid in screening individuals at risk of developing CRC. In addition, there remains the possibility that other MYH mutations common in the Australian population may indeed modify colorectal cancer risk in an otherwise high-risk population.

\section{References}

1. Lynch HT and Smyrk T. Hereditary nonpolyposis colorectal cancer (Lynch syndrome). An updated review. Cancer 1996; 78 (6): $1149-1167$.

2. Kinzler KW and Vogelstein B. Lessons from hereditary colorectal cancer. Cell 1996; 87 (2): 159-170.

3. Aaltonen LA, Salovaara R, Kristo P, Canzian F, Hemminki A, Peltomaki P, Chadwick RB, Kaariainen H, Eskelinen M, Jarvinen $\mathrm{H}$, Mecklin JP and de la Chapelle A. Incidence of hereditary nonpolyposis colorectal cancer and the feasibility of molecular screening for the disease. N Engl J Med 1998; 338: 1481-1487.

4. Peltomaki P, Gao P and Mecklin JP. Genotype and phenotype in hereditary nonpolyposis colon cancer: a study of families with different vs. shared predisposing mutations. Fam Cancer 2001; 1 (1): 9-15.

5. Lynch HT and de la Chapelle A. Genetic susceptibility to non-polyposis colorectal cancer. J Med Genet 1998; 36 (11): 801-818.

6. Watson $\mathrm{P}$ and Lynch HT. Cancer risk in mismatch repair gene mutation carriers. Fam Cancer 2001; 1 (1): 57-60.

7. Mitchell RJ, Farrington SM, Dunlop MG and Campbell H. Mismatch repair genes $\mathrm{hMLH} 1$ and $\mathrm{hMSH} 2$ and colorectal cancer: a HuGE review. Am J Epidemiol 2002; 156 (10): 885-902.

8. Riicken FE, Mourits MJ, Kleibeuker JH, Hollema H and van der Zee AG. Gynaecologic screening in hereditary nonpolyposis colorectal cancer. Gynaecol Oncol 2003; 91 (1): 74-80.

9. Nakabeppu Y, Tsuchimoto D, Furuichi M and Sakumi K. The defensive mechanisms in mammalian cells against oxidative damage in nucleic acids and their involvement in the suppression of mutagenesis and cell death. Free Rad Res 2004; 38: 423-429.

10. Al-Tassan N, Chmiel NH, Maynard J, Fleming N, Livingston AL, Williams GT, Hodges AK, Davies DR, David SS, Sampson JR and Cheadle JP. Inherited variants of MYH associated with somatic G: C->T: A mutations in colorectal tumors. Nat Genet 2002; 30: 227-232.

11. Kairupan CF, Meldrum CM, Crooks R, Milward EA, Spiegelman AD, Burgess B, Groombridge C, Kirk J, Tucker K, Ward R, Williams R and Scott RJ. Mutation analysis of the MYH gene in an Australian series of colorectal polyposis patients with or without germline APC mutations. Int J Cancer (epub 10 Mar 2005).

12. Bai H, Jones S, Guan X, Wilson TM, Sampson JR, Cheadle JP and Lu A. Functional characterization of two human MutY homolog (hMYH) missense mutations (R227W and V232F) that lie within the putative hMSH6 binding domain and are associated with hMYH polyposis. Nucleic Acids Res 2005; 33 (2): 597-604.

13. Kambara T, Whitehall VLJ, Spring KJ, Barker MA, Arnold S, Wynter CVA, Matsubara N, Tanaka N, Young JP, Leggett BA and Jass JR. Role of inherited defects of MYH in the development of sporadic colorectal cancer. Gen Chrom\&Can 2004; 40 (1): 1-9.

14. Croitoru ME, Cleary SP, Di Nicola N, Manno M, Selander T, Aronson M, Redston M, Cotterchio M, Knight J, Gryfe R and Gallinger S. Association between biallelic and monoallelic germline MYH gene mutations and colorectal cancer risk. J Natl Cancer Inst 2004; 96 (21): 1631-1634.

15. Jones S, Emmerson P, Maynard J, Best JM, Jordan S, Williams GT, Sampson JR and Cheadle JP. Biallelic germline mutations in MYH predispose to multiple colorectal adenoma and somatic G: C->T: A mutations. Hum Mol Genet 2002; 23: 2961-2967. 
16. Gismondi V, Meta M, Bonelli L, Radice P, Sala P, Bertario L, Viel A, Fornasarig M, Arrigoni A, Gentile M, Ponz de Leon M, Anselmi L, Mareni C, Bruzzi P and Varesco L. Prevalence of the Y165C, G382D and 1395delGGA germline mutations of the MYH gene in Italian patients with adenomatous polyposis coli and colorectal adenomas. Int J Cancer 2004; 109: 680-684.

17. Sieber OM, Lipton L, Crabtree M, Heinimann K, Fidalgo P, Phillips RK, Bisgaard ML, Orntoft TF, Aaltonen LA, Hodgson SV, Thomas HJ and Tomlinson IP. Multiple colorectal adenomas, classic adenomatous polyposis, and germline mutations in $\mathrm{MYH}$. N Engl J Med 2003; 348: 791-799.

18. Gu Y, Parker A, Wilson TM, Bai H, Chang D and Lu A. Human MutY homolog, a DNA glycosylase involved in base excision repair, physically and functionally interacts with mismatch repair proteins human MutS homolog 2/human MutS homolog 6. J Biol Chem 2002; 277 (13): $11135-11142$.

19. Miller SA, Dykes DD and Polesky HF. A salting out procedure for extracting DNA from human nucleated cells. Nucleic Acids Res 1988; 16: 739.

20. Kemp Z, Thirlwell C, Sieber O, Silver A and Tomlinson I. An update on the genetic of colorectal cancer. Hum Mol Genet 2004; 13 Suppl 2: R177-185.

21. Parker A, Gu Y, Mahoney W, Lee SH, Singh KK and Lu AL. Human homolog of the MutY repair protein (hMYH) physically interacts with proteins involved in long patch DNA base excision repair. J Biol Chem 2001; 276: 5547-5555.

22. Shinmura K, Yamaguchi S, Saitoh T, Kohno T and Yokota J. Somatic mutations and single nucleotide polymorphisms of base excision repair genes involved in the repair of 8-hydroxyguanine in damaged DNA. Cancer Lett 2001; 166: 65-69. 